\title{
A Multi-scale Parallel Numerical Solver for Modeling of Two-phase Viscoelastic Fluids Based on the OpenFOAM
}

\author{
Xiao-Wei Guo* \\ State Key Laboratory of High Performance Computing \\ National University of Defense Technology \\ Changsha, China \\ *Corresponding author
}

\author{
Yu Cao, Miao Wang, and Qian Wang \\ State Key Laboratory of High Performance Computing \\ National University of Defense Technology \\ Changsha, China
}

\begin{abstract}
With the rapid development of high performance computing technology, the simulation of viscoelastic fluids has become an extremely important research area and numerous new promising techniques have been proposed over the last decades. In this paper we proposed a numerical algorithm for solving the multi-scale two-fluid model, additionally, based on an open source CFD toolbox, we implemented a parallel solver and verified the algorithms through parallel simulations. The results verified the numerical algorithm and show that the parallel codes of the solver have good parallel efficiency.
\end{abstract}

Keywords-numerical simulation; multi-scale; OpenFOAM; parallel computing; viscoelastic fluids

\section{INTRODUCTION}

Viscoelastiticy refers to the property of materials that exhibit both viscous and elastic characteristics when undergoing deformation. Many natural and synthetic fluids exhibit viscoelastic characteristics; notable examples include blood, DNA solutions, polymer melts and solutions, and fiber suspensions [1-4]. The most challenging part of modeling the viscoelastic fluids is to determine the non-linear coupling between the macroscopic rheological responses and the microscopic evolution of molecular configurations under flow, thus modeling the viscoelastic fluids intrinsically is a multiscale problem.

As a microscopic approach, the atomistic modeling is limited to the flow geometries of molecular dimensions due to the massive computing resource requirements. Recent years, the coarse-grained molecular kinetic theory gained major devolvement, and numerous constitutive equations derived from the closure approximations of a kinetic theory were proposed [5]. Nevertheless, the approximations such as the preaveraging or the decoupling involved for a macroscopic constitutive equation significantly impact on the rheological predictions of viscoelastic fluids. The micro-macro methods that couple the macroscopic continuum mechanics with the microscopic coarse-grained molecular kinetic theory plays a much more important role in the simulation of viscoelastic fluids. The Brownian Configuration Field (BCF) method [6] is a new promising micro-macro simulation approach for the complex fluids simulations.
In this paper, we aim to numerically solve a multi-scale two-fluid model based on the BCF approach. The detailed algorithms are described along with the parallel implementation based on an open source CFD toolbox, named OpenFOAM [7]. The main contributions of the paper are as follows:

- Proposed a numerical algorithm for solving the multiscale two-fluid model;

- Implemented a parallel solver for modeling of twophase viscoelastic fluids based on the OpenFOAM;

- Verified the numerical solver and the algorithms through parallel simulations.

The remainder of this article is organized as follows: Firstly we give the basic ideas and describe the architecture of the numerical solver in Section II. In Section III the governing equations for modeling the two-phase viscoelastic fluids are presented and followed by the iterative algorithm to solve the model. The simulation results are showed in Section IV. Some related work in viscoelastic fluids simulation is reviewed in Section V. We present our conclusions in Section VI.

\section{THE ARCHITECTURE OF THE NUMERICAL SOLVER BASED ON OPENFOAM}

To discretize the governing equations and solve the discretized linear systems, we use an Open Source CFD toolbox released by the OpenCFD Ltd, named OpenFOAM. OpenFOAM is a $\mathrm{C}++$ library used to solve the CFD problems. Many typical linear system solvers and discretization schemes are predefined in this library. Appropriate type of solvers and the schemes could be configured through the control dictionary file. The OpenFOAM uses programming languages that is very close to the verbal and mathematical languages used in science and engineering, thus writing a numerical solver based on OpenFOAM becomes significantly easier. The schematic diagram of the architecture for written a numerical solver is shown in Figure I. It forms a four-layer structure to keep the key functions of the numerical solver independent. The iterative numerical algorithms are written in OpenFOAM programming languages in the Multi-scale Numerical solver layer. 


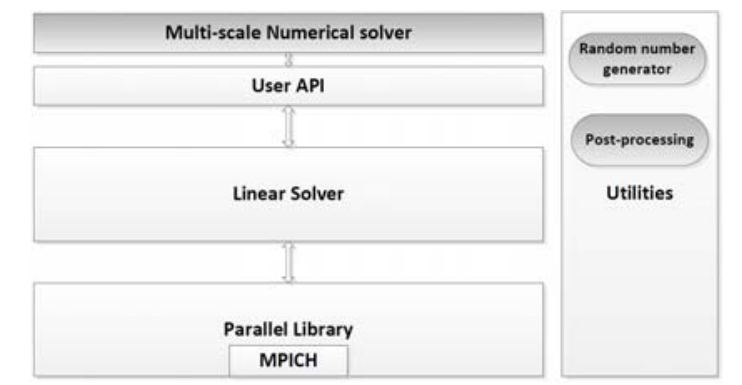

FIGURE I THE SCHEMATIC DIAGRAM OF THE PARALLEL NUMERICAL SOLVER BASED ON OPENFOAM

The User API layer provides all the necessary classes and interfaces to write the equations in $\mathrm{C}++$. At this point, the users do not need to know anything about the linear system solvers and the parallel computing techniques in detail. The application to describe the Algorithm 1 can be written in OpenFOAM programming languages. The Linear solver layer contains numerous most commonly used linear equation algorithms including the conjugate (PCG) and bi-conjugate gradient (PBiCG) methods. The discretization schemes used in the numerical solver are also implemented in this layer and the configurations used in our solver are listed in Table I.

TABLE I THE DISCRETISATION SCHEMES USED IN THE NUMERICAL SOLVER FOR DIFFERENT TERMS

\begin{tabular}{ccc}
\hline Term & Scheme & Order of accuracy \\
\hline Temporal term & Euler & First order \\
Gradient term & Gauss linear & Second order \\
Divergence term & Gauss linear & Second order \\
Laplacian term & Gauss linear corrected & Second order \\
Convection term default & Gauss Minmod & Second order \\
Convection term for $\phi_{A}$ & Gauss vanLeer & First order \\
\hline
\end{tabular}

In this architecture, the only module related to the parallel communication is the Parallel Library layer. Here the MPICH library is used and the interfaces provided in this layer can enable the leaner solvers communicating with each other while running in parallel.

\section{The Numerical Algorithm For Modeling Two- PHASE VISCOELASTIC FLUIDS}

\section{A. The Governing Equations of A Multi-scale Two-fluid Model}

To model the phase transitions of a two-phase viscoelastic fluid, macroscopic constitutive equations are replaced to the BCF approach for calculating the viscoelastic stress tensor. The governing equations for the full model are brought together below.

For isothermal and incompressible viscoelastic fluids with density, the continuity equation and the momentum balance equation can be expressed as

$$
\vec{\nabla} \cdot \vec{v}=0
$$

and

$$
\rho \frac{D \vec{v}}{D t}=\eta_{s} \nabla^{2} \vec{v}-\vec{\nabla} p-\left(2 \phi_{A}-1\right) \vec{\nabla} \mu+\vec{\nabla} \cdot \sigma_{\mathrm{p}}(\vec{r}, t)
$$

The evolution equation of the composition field A could be written as

$$
\frac{D \phi_{A}(\vec{r}: t)}{D t}=\vec{\nabla} \cdot\left[\frac{\phi_{A}^{2}\left(1-\phi_{A}\right)^{2}}{\varsigma}\left(\vec{\nabla} \mu-\alpha \vec{\nabla} \cdot \sigma_{\mathrm{p}}\right)\right]
$$

The thermodynamic effects in two-fluid framework are described through the chemical potential difference $\mu=\mu_{A}-\mu_{B}$, which could be defined as the functional derivative of the mixing free energy with respect to local volume fraction, i.e.,

$$
\mu=\frac{\delta \mathcal{F}_{\text {mix }}\left[\phi_{A}(\vec{r})\right]}{\delta \phi_{A}(\vec{r})}
$$

We take a first order approximation of the Flory-Hugginsde-Gennes form for the mixing free energy function $\mathrm{F}_{\text {mix }}$.

The viscoelastic stress tensor $\sigma_{p}$ is considered on a microscopic viewpoint. To simulating the distribution of the molecular chain, $N_{f}$ configuration fields $\vec{Q}_{i}(\vec{r}, t)$ are introduced to replace the Fokker-Planck equation, therefore the corresponding stochastic differential equation can be rewritten as [6]

$$
\begin{aligned}
& d \vec{Q}_{i}(\vec{r}, t)=\left[-\vec{v}(\vec{r}, t) \cdot \nabla \vec{Q}_{i}(\vec{r}, t)+(\nabla \vec{v}(\vec{r}, t))^{T} \cdot \vec{Q}_{i}(\vec{r}, t)\right) \\
& \left.-\frac{F\left(\vec{Q}_{i}(\vec{r}, t)\right)}{2 \lambda\left(\phi_{A}\right)}\right] d t+\sqrt{\frac{1}{\lambda\left(\phi_{A}\right)}} d \vec{W}_{i}(t)
\end{aligned}
$$

According to the Kramer's expression, the viscoelastic stress $\sigma_{p}$ can be given by [8]

$$
\sigma_{\mathrm{p}}=\left(\frac{b+d+2}{b}\right) \frac{\eta_{p}\left(\phi_{A}\right)}{\lambda\left(\phi_{A}\right)}(\langle\vec{Q} \otimes F(\vec{Q})\rangle-I)
$$

and

$$
\tilde{\mathrm{Q}} \oplus \mathrm{F}(\tilde{\mathrm{Q}})^{\mathrm{E}}{ }^{1 / 4}{ }_{i=1}^{\mathrm{P}_{f}} \tilde{Q}_{i}(\tilde{r} ; t) \oplus \mathrm{F}\left(\tilde{Q}_{i}(\tilde{r} ; t)\right)
$$

In this representation $\mathrm{dW}_{\mathrm{i}}(\mathrm{t})$ only depends on time and essentially are independent Gaussian variables with zero mean and variance $\mathrm{dt}$.

\section{B. An Iterative Numerical Algorithm Based on OpenFOAM}

To numerically solve the multi-scale model described in Section III, a modified PISO iterative algorithm is adopted. In our previous research, A similar algorithm for solving the macroscopic model has been well tested in a numerical study for the dynamics of polymer solutions in contraction flow [9]. Additionally, the shear-banding flows with a macroscopic twofluid model have been studied recently [10]. The revised version of the multi-scale numerical algorithm has been presented in Algorithm 1. In this algorithm, steps $4 \sim 7$ solved the microscopic equations to obtain the viscoelastic stress. The modified PISO iterations presented in steps 8 11, then followed by the volume fraction calculations at steps $12 \sim 14$. It should be noted that the correction steps $13 \sim 14$ are added to restrict the volume fraction variables in the range of $[0,1]$. 
Data: Mesh data, initial conditions

Result: $\vec{v}, p, \sigma_{\mathrm{p}}, \phi_{A}$

1 read the mesh data and the initial conditions;

2 initialization;

3 while $t^{n+1}$ not reach the end of the simulation time do for $i=1$ to $N_{f}$ do

$5 \quad \mid$ Solve Eq.(5) to get the configuration field $\vec{Q}_{i}(\vec{r}, t)$ at time $t^{n+1}$ using a semi-implicit Euler method: $\left(1+\frac{\Delta t^{n}}{2 \lambda\left(\phi_{A}^{n}\right)\left(1-\left|Q_{i}^{n+1}(\vec{r})\right|^{2} / b\right)}\right) Q_{i}^{\overrightarrow{n+1}}(\vec{r})=$ $\overrightarrow{Q_{i}^{n}}(\vec{r})+\left[-v^{\vec{n}}(\vec{r}) \cdot \nabla \overrightarrow{Q_{i}^{n}}(\vec{r})+\left(\nabla \overrightarrow{v^{n}}(\vec{r})\right)^{T}\right.$. $\left.\left.\overrightarrow{Q_{i}^{n}}(\vec{r}, t)\right)\right] \Delta t^{n}+\sqrt{\frac{\Delta t^{n}}{\lambda\left(\phi_{A}^{n}\right)}} \vec{N}_{i}(0,1)$;

end

7 Compute the polymer stress tensor using Kramers' expression $\sigma_{\mathrm{p}}^{n+1}(\vec{r})=$

$\left(\frac{b+d+2}{b}\right) \frac{\eta_{p}\left(\phi_{A}^{n}\right)}{\lambda\left(\phi_{A}^{n}\right)}\left(\sum_{i=1}^{N_{f}} Q_{i}^{\overrightarrow{n+1}}(\vec{r}) \otimes F\left(Q_{i}^{\overrightarrow{n+1}}(\vec{r})\right)-\boldsymbol{I}\right) ;$

8

Solve the discretised momentum equation

$\frac{\partial \vec{v}}{\partial t}+\vec{v} \cdot \vec{\nabla} \vec{v}-\frac{\eta_{A}}{\rho} \nabla^{2} \vec{v}+\frac{\left(2 \phi_{A}-1\right)}{\rho} \vec{\nabla} \mu-\frac{\vec{\nabla} \cdot \sigma_{\mathrm{p}}(\vec{r}, t)}{\rho}=-\frac{\vec{\nabla} p}{\rho}$ to obtain the estimated components $\vec{U}^{(n+1) *}$;

9 Solve the pressure-correction equation $\nabla \cdot \vec{U}^{n+1}=\nabla \cdot\left(A^{-1} \nabla[p]\right)^{n+1}$ to obtain the pressure field $p^{(n+1) *}$;

10 Calculate the corrected velocity field $\vec{v}^{(n+1) *}$ by solving $\vec{v}^{n+1}=\vec{U}^{n+1}-A^{-1} \nabla[p]^{n}$;

11 Repeat the steps $8 \sim \mathbf{1 0}$ using the corrected $p^{(n+1) *}, \vec{v}^{(n+1) *}$ until all corrections are negligibly small for the solutions at the present time $\vec{v}^{n+1}$ and $p^{n+1}$;

12 Solve Eq.(3) using $\sigma_{\mathrm{p}}^{n+1}$ and $\vec{v}^{n+1}$ to obtain the volume fraction $\phi_{A}^{n+1}$;

13 Solve the equation for $\phi_{B}$ by replacing $\phi_{A}=1-\phi_{B}$ to get $\phi_{B}^{n+1}$;

14 Volume fraction correction: $\phi_{A}=\frac{\phi_{A}}{\phi_{A}+\phi_{B}}$, $\phi_{B}=1-\phi_{A}$

$15 \quad n \leftarrow n+1$;

16 end

Algorithm 1 The iterative algorithm to solve the multi-scale two-fluid model

\section{Simulation Results}

The codes of the parallel solver have been investigated in test simulations on a HPC cluster located in the State Key Laboratory of High Performance Computing. Each computing node of this cluster contains 12 Intel Xeon E5-2620 2.10GHz CPU cores and a total main memory of $16 \mathrm{~GB}$. The parallel simulations are executed by distributed the processes to the computing nodes with 1 process every CPU core.

The main parameters of the multi-scale model remain constant throughout all of the simulations and are set as shown in Table II. Furthermore, we specify a poiseuille flow in a planar channel as shown in Figure II. In a planar channel, the viscoelastic fluid flows driven by the constant pressure gradient between the inlet and the outlet of the channel.
TABLE II PARAMETERS USED IN SIMULATIONS

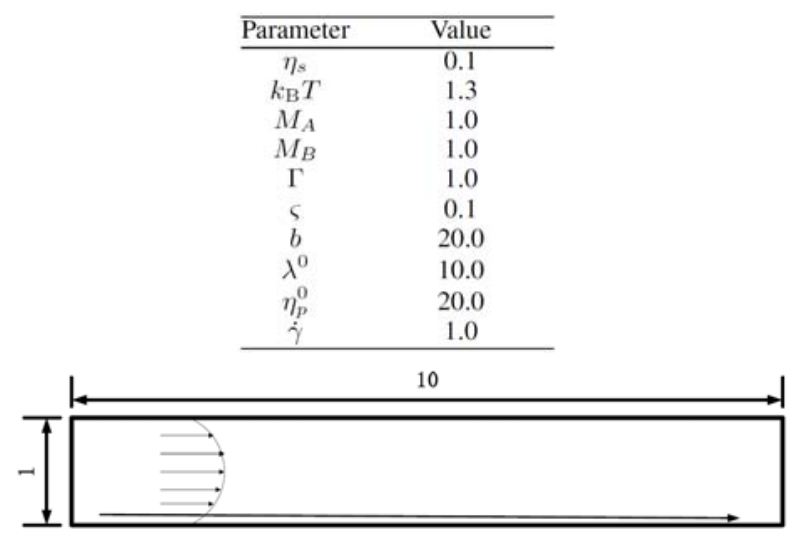

FIGURE II THE POISEUILLE FLOW IN A PLANAR CHANNEL WITH

THE LENGTH RATIO $\frac{1_{\mathrm{x}}}{1_{\mathrm{y}}}=10$. THE FLOW FIELD IS DRIVEN BY OCCURRING CONSTANT PRESSURE GRADIENT IN THE CHANNEL DIRECTION.

The steady-state velocity $U_{\mathrm{x}}$ and the corresponding viscoelastic stress component $1 / 4 \times \mathrm{x}$ are sampled after a long time simulation with $\mathrm{T}_{\mathrm{sim}}>100$. At a fixed $\mathrm{x}$-axis $\mathrm{x}=5.0$, the profiles are plotted in Figure III. The parabolic profiles of the simulation results are consistent with previous research.

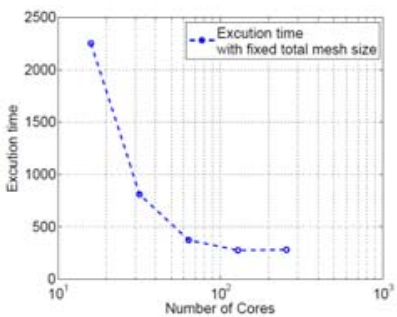

(a)

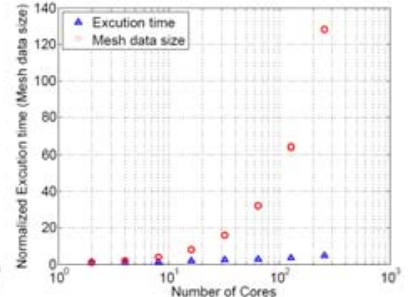

(b)
FIGURE III (A) THE STRONG SCALABILITY WITH FIXED TOTAL MESH SIZE 1048576; (B) THE WEAK SCALABILITY FOR FIXED MESH SIZE 8192 PER PROCESSOR CORE

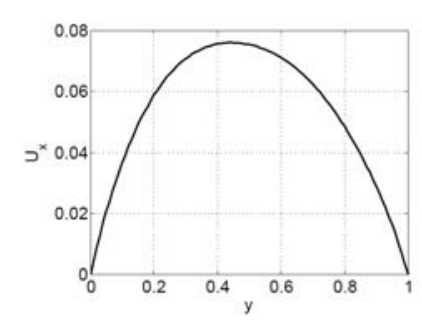

(a) $\vec{v}_{x}$

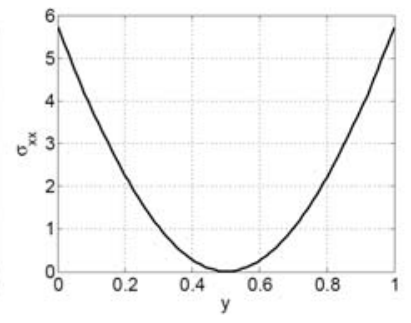

(b) $\sigma_{x x}$
FIGURE IV THE STEADY-STATE VELOCITY FIELD $\mathrm{U}_{\mathrm{x}}$ AND THE VISCOELASTIC STRESS COMPONENT ${ }^{3 / 4} \times x^{2}$ AT A FIXED X-AXIS X = 5.0

To evaluate the scalability of the parallel codes based on the OpenFOAM, firstly we fixed the problem size with a mesh of 1048576 cells, and the execution times varies with the number of processor cores are plotted in Figure IV(a). From 16 to 128 processor cores, the time cost is significantly reduced as the 
parallel scale increasing. Nevertheless, the execution time cannot be decreased as the core number larger than 128. As shown in the plot, the execution time with 128 cores and 256 cores are almost the same. It is found that the optimal number of mesh cells for each processor core may be around 8192 . Therefore a fixed mesh size of 8192 cells are distributed to each core in the weak scalability tests as presented in Figure IV(b). As the parallel scale (processor cores) raising, the total problem size measured by the mesh data size increases linearly, however the execution time of the simulations changes little. That means the parallel codes implemented in this paper can be easily scale to more than 256 processor cores.

\section{RELATED WORK}

With the developing of the high performance computing technology, the simulation of viscoelastic fluids has become an extremely important research area and numerous new promising techniques have been proposed over the last decades. The atomistic modeling is the most detailed approach to describe the rheological behavior in complex fluids, however, considering the massive computer resource requirement, this microscopic approach is limited to flow geometries of molecular dimensions. Thus some micro-macro methods [5] were introduced that coupled the coarse-grained molecular kinetic theory to the macroscopic continuum equations. The Brownian configuration field (BCF) method proposed by Hulsen et al.[6] is a promising new multi-scale approach to model the viscoelastic fluids. The key idea of the BCF method is using Brownian configuration fields instead of tracking discrete particles, and that significantly reduces the drawbacks of the CONNFFESSIT method introduced by Laso and Ottinger[11].

In practice the BCF method works very well and has been applied to simulation of numerous viscoelastic flows, including the flow past a cylinder[6,12], viscoelastic free surface flows[13], contraction and expansion flows[14], Couette flow, Poiseuille flow, lid driven cavity flow[8] and flow between eccentrically rotating cylinders [15]. Due to the spatial smoothness, the BCF method has a considerable increased numerical stability. This advantage was confirmed in previous research and Mangoubi [16] recently gave an in-depth analysis about the origin of the numerical stability of the BCF method.

The numerical solver proposed in this paper is inspired by these previous researches. We expect the research will motivate more progress in large-scale parallel simulation for viscoelastic fluids.

\section{CONCLUSION}

The dynamics under flow of multi-phase viscoelastic fluids plays essential roles in numerous applications ranging from biological systems to the industrial production. As modeling the two-phase viscoelastic fluids is a multi-scale problem, we introduce a macro-micro model that couples the microscopic BCF method into the macroscopic two-fluid framework. In this paper, we give a parallel numerical algorithm to solve this multi-scale two-fluid model, and the basic ideas to implement the solver based on an open source CFD toolbox have been presented in detail. Finally, the parallel codes of the numerical solver have been tested in a HPC cluster. The profile of the simulation results and the parallel scalability are presented. The results verified the numerical algorithm and show that the solver has good parallel efficiency.

\section{ACKNOWLEDGMENT}

The authors would like to thank the National Natural Science Foundation of China (Grant No.61221491, No.61303071 and No. 61120106005), and the Open fund from HPCL (No. 201303-01, 201503-01 and 201503-02) for funding.

\section{REFERENCES}

[1] H. Tanaka, "Viscoelastic phase separation in soft matter and foods," Faraday Discussions, vol. 158, pp. 371-406, 2012

[2] J. Sprakel, E. Spruijt, M. A. C. Stuart, N. A. M. Besseling, M. P. Lettinga, and J. van der Gucht, "Shear banding and rheochaos in associative polymer networks," Soft Matter, vol. 4, no. 8, pp. 16961705, 2008.

[3] B. Lonetti, M. Camargo, J. Stellbrink, C. N. Likos, E. Zaccarelli, L. Willner, P. Lindner, and D. Richter, "Ultrasoft colloid-polymer mixtures: Structure and phase diagram,” Physical Review Letters, vol. 106, no. 22, p. 228301, 2011.

[4] P. E. Boukany and S. Q. Wang, "Shear banding or not in entangled dnasolutions,” Macromolecules, vol. 43, no. 17, pp. 6950-6952, 2010.

[5] R. Keunings, "Micro-macro methods for the multiscale simulation of viscoelastic flow using molecular models of kinetic theory,” Rheology reviews, vol. 2004, pp. 67-98, 2004.

[6] M. Hulsen, A. Van Heel, and B. Van Den Brule, "Simulation of viscoelastic flows using brownian configuration fields," Journal of NonNewtonian Fluid Mechanics, vol. 70, no. 1, pp. 79-101, 1997.

[7] The OpenFOAM user guide, 2nd ed., The OpenFOAM Foundation, 32014. [Online]. Available: http://www.openfoam.org/docs/

[8] X. Y. Xu, J. Ouyang, W. M. Li, and Q. S. Liu, "Sph simulations of 2d transient viscoelastic flows using brownian configuration fields,” Journal of Non-Newtonian Fluid Mechanics, vol. 208, pp. 59-71, 2014.

[9] S. C. Omowunmi and X. F. Yuan, "Time-dependent non-linear dynamics of polymer solutions in microfluidic contraction flow-a numerical study on the role of elongational viscosity,” Rheologica Acta, vol. 52, no. 4, pp. 337-354, 2013.

[10] X.-W. Guo, S. Zou, X. Yang, X.-F. Yuan, and M. Wang, “Interface instabilities and chaotic rheological responses in binary polymer mixtures under shear flow,” RSC Advances, vol. 4, no. 105, pp. 61 16761 177, 2014.

[11] H. ttinger, B. Van Den Brule, and M. Hulsen, "Brownian configuration fields and variance reduced connffessit,” Journal of non-newtonian fluid mechanics, vol. 70, no. 3, pp. 255-261, 1997.

[12] X. J. Fan, N. Phan-Thien, and R. Zheng, "Simulation of fibre suspension flows by the brownian configuration field method," Journal of NonNewtonian Fluid Mechanics, vol. 84, no. 23, pp. 257-274, 1999.

[13] M. Bajaj, P. P. Bhat, J. R. Prakash, and M. Pasquali, "Multiscale simulation of viscoelastic free surface flows," Journal of Non-Newtonian Fluid Mechanics, vol. 140, no. 1-3, pp. 87-107, 2006.

[14] Z. M. Lu, B. C. Khoo, H. S. Dou, N. Phan-Thien, and K. S. Yeo, "Numerical simulation of fibre suspension flow through an axisymmetric contraction and expansion passages by brownian configuration field method," Chemical Engineering Science, vol. 61, no. 15, pp. 4998-5009, 2006.

[15] T. N. Phillips and K. D. Smith, "A spectral element approach to the simulation of viscoelastic flows using brownian configuration fields," Journal of Non-Newtonian Fluid Mechanics, vol. 138, no. 2-3, pp. 98110, 2006.

[16] C. Mangoubi, M. A. Hulsen, and R. Kupferman, "Numerical stability of the method of brownian configuration fields," Journal of NonNewtonian Fluid Mechanics, vol. 157, no. 3, pp. 188-196, 2009. 